\title{
Isolated Limb Infusion and Isolated Limb Perfusion for Melanoma: Can the Outcomes of these Procedures be Compared?
}

\author{
Hidde M. Kroon, MD, PhD ${ }^{1,2}$ and John F. Thompson, MD, FRACS, FACS ${ }^{1,3,4}$ \\ ${ }^{1}$ Melanoma Institute Australia, The University of Sydney, North Sydney, NSW,Australia; ${ }^{2}$ Department of General Surgery, \\ The University of Adelaide, Royal Adelaide Hospital, Adelaide, SA, Australia; ${ }^{3}$ Discipline of Surgery, The University of \\ Sydney, Sydney, NSW, Australia; ${ }^{4}$ Department of Melanoma and Surgical Oncology, Royal Prince Alfred Hospital, \\ Sydney, NSW, Australia
}

When bulky or numerous local melanoma recurrences or in-transit melanoma metastases develop in a limb, simple local treatment modalities such as excision, cryotherapy, laser ablation, or intralesional injection with Rose Bengal (PV-10) or talimogene laherparepvec (T-VEC) are likely to be ineffective or simply not possible. ${ }^{1}$ However, achieving adequate disease control is important in these patients as the metastatic lesions frequently cause troublesome symptoms such as pain and ulceration with oozing, odor, and hemorrhage, sometimes accompanied by restricted mobility and social isolation. ${ }^{2}$ Moreover, those who develop extensive recurrent disease are often elderly, many of them physically frail and suffering from multiple comorbidities. $^{2}$ Treatment of these patients is therefore aimed at achieving effective disease control and, most importantly, limb preservation, using a treatment with limited locoregional toxicity that is unlikely to cause serious systemic side effects.

Traditionally, a single isolated limb perfusion (ILP) or, more recently, the minimally invasive alternative of a single isolated limb infusion (ILI) is performed in these cases. ${ }^{3}$ Both procedures are effective and, even in this modern era of more satisfactory systemic therapies for metastatic melanoma, they are still worthwhile and relatively straightforward, single-treatment options to treat

(C) Society of Surgical Oncology 2018

First Received: 28 August 2018;

Published Online: 21 November 2018

J. F. Thompson, MD, FRACS, FACS

e-mail: John.Thompson@melanoma.org.au locally recurrent or in-transit metastatic melanoma involving a limb. ${ }^{4}$ Furthermore, both procedures have acceptable, usually minor, locoregional postoperative toxicity with a short hospital stay. ${ }^{3}$ Overall responses of 64-96\% can be achieved after ILP, while, in large ILI series, overall response rates of $43-84 \%$ have been reported, with somewhat lower complete response rates of 27-44\% after ILI than those reported after ILP $(25-69 \%){ }^{5-9}$ Although the reported response rates after ILP are slightly higher, no statistically valid comparison of the two procedures by means of a randomized controlled trial or, alternatively, a case-control study allowing similar patient populations to be compared, has been performed to date.

In recent years, several articles have been published comparing ILI and ILP as a single-center experience or as a two-center experience in historical cohorts of patients. ${ }^{7,8}$ In other articles, ILP outcomes have been analyzed and compared with the results of ILI reported in the literature. ${ }^{5}$ Most of these studies reported outcomes in favor of ILP, with higher complete response rates, and concluded that ILP is a more effective procedure. We suggest that this conclusion may not be appropriate. Since the patient populations treated by the two procedures are actually not similar, reported outcomes of ILI and ILP cannot simply be compared. ${ }^{2}$ Most patients in ILI studies were older and had a higher stage of disease, both of which are predictive factors for poorer outcome. ${ }^{2,7,8,10}$ Bearing in mind these differences in baseline patient characteristics, lower response rates after ILI were to be expected. It is therefore likely that compared with ILI studies, patient selection for ILP may have also contributed to the better outcomes. 
Moreover, looking only at response rates following regional therapies such as ILI and ILP may actually not be the most accurate way of defining efficacy in these elderly and frail patients.

The reason that surgeons tend to offer ILI to older, medically frail patients suffering from higher stages of disease is likely because the procedure is well-tolerated, making it feasible to treat many of them who would have been considered unsuitable for treatment by ILP, a more invasive, more prolonged, and complex procedure. ${ }^{2,3}$ The morbidity after ILI is markedly lower than after ILP, which more often results in wound infections, postoperative bleeding, or compartment syndromes. ${ }^{11,12}$ Amputation due to severe regional toxicity is extremely rare after melphalan ILI and to date has only been reported once, whereas after ILP this has been reported in $0.65-3.2 \%$ of patients. ${ }^{6,7,9}$ Lastly, while mortality has not been reported following ILI, multiple studies have mentioned post-procedural mortality following ILP. ${ }^{2,5,13}$ Also to be considered is the fact that systemic side effects are frequently observed following ILP due to leakage of cytotoxic drugs into the systemic circulation because of the high intravascular pressure and high volume flow that are maintained in the isolated limb. Following ILI, as a result of the low-flow circulation, leakage to the systemic circulation is negligible and systemic side effects are rare. ${ }^{11,14}$

In this new era of effective systemic therapies and novel local treatment options, the indications for ILI and ILP as independent treatment modalities are becoming more limited and they are increasingly likely to play a role as part of combined therapy for patients with locoregional melanoma metastases. This approach was used in a recent study by Ariyan et al. ${ }^{15}$ using systemic ipilimumab in combination with melphalan ILI in patients with stage IIIB-IV disease; an overall response rate of $85 \%$ was achieved (complete response rate $62 \%$ ). In view of this emerging treatment paradigm, a minimally invasive procedure such as ILI would be preferred, allowing the patient to recover more quickly and with a lower risk of complications, so as not to interfere with concurrent or immediately subsequent systemic treatment. ${ }^{4,9,15}$

In conclusion, it is likely that it is not only the ILP procedure itself but also the patients selected for the procedure that lead to somewhat higher response rates than those achieved by ILI. However, these reported differences in response rates have to be interpreted with great caution, and allowances made for age, comorbidities, and stage of disease before firm conclusions can be drawn about the efficacy of the two forms of locoregional therapy. In view of the higher morbidity following the more labor-intensive ILP, and with the likelihood of future systemic treatment modalities being combined with regional therapies, assessments based solely on response rates may not be the optimal method of defining their value in elderly and frail patients. Ultimately, a randomized controlled trial comparing ILP and ILI directly will be required for a valid comparison of the two procedures.

\section{REFERENCES}

1. Testori A, Faries MB, Thompson JF, et al. Local and intralesional therapy of in-transit melanoma metastases. J Surg Oncol. 2011;104:391-6.

2. Kroon HM, Coventry BJ, Giles MH, et al. Safety and efficacy of isolated limb infusion chemotherapy for advanced locoregional melanoma in elderly patients: an Australian multicenter study. Ann Surg Oncol. 2017;24:3245-51.

3. Sanki A, Kroon HM, Kam PC, Thompson JF. Isolated limb perfusion and isolated limb infusion for malignant lesions of the extremities. Curr Probl Surg. 2011;48:371-430.

4. Grünhagen DJ, Kroon HM, Verhoef C. Perfusion and infusion for melanoma in-transit metastases in the era of effective systemic therapy. Am Soc Clin Oncol Educ Book. 2015;35:e528-34.

5. Madu MF, Deken MM, van der Hage JA, Jóźwiak K, Wouters MW, van Akkooi AC. Isolated limb perfusion for melanoma is safe and effective in elderly patients. Ann Surg Oncol. 2017;24:1997-2005.

6. Moreno-Ramirez D, de la Cruz-Merino L, Ferrandiz L, VillegasPortero R, Nieto-Garcia A. Isolated limb perfusion for malignant melanoma: systematic review on effectiveness and safety. Oncologist. 2010;15:416-27.

7. Raymond AK, Beasley GM, Broadwater G, et al. Current trends in regional therapy for melanoma: lessons learned from 225 regional chemotherapy treatments between 1995 and 2010 at a single institution. J Am Coll Surg. 2011;213:306-16.

8. Dossett LA, Ben-Shabat I, Olofsson Bagge R, Zager JS. Clinical response and regional toxicity following isolated limb infusion compared with isolated limb perfusion for in-transit melanoma. Ann Surg Oncol. 2016;23:2330-5.

9. Kroon HM, Huismans AM, Coventry BJ, Thompson JF. Isolated limb infusion for recurrent and locally metastatic limb melanoma. In: Riker A, editors. Melanoma. New York: Springer; 2018. pp. 451-65.

10. Kroon HM, Moncrieff M, Kam PC, Thompson JF. Outcomes following isolated limb infusion. A 14-year experience. Ann Surg Oncol. 2008;15:3003-13.

11. Möller MG, Lewis JM, Dessureault S, Zager JS. Toxicities associated with hyperthermic isolated limb perfusion and isolated limb infusion in the treatment of melanoma and sarcoma. Int $J$ Hyperthermia. 2008;24:275-89.

12. Beasley GM, Petersen RP, Yoo J, et al. Isolated limb infusion for in-transit malignant melanoma of the extremity: a well-tolerated but less effective alternative to hyperthermic isolated limb perfusion. Ann Surg Oncol. 2008;15:2195-205.

13. Van Etten B, van Geel AN, de Wilt JH, et al. Fifty tumor necrosis factorbased isolated limb perfusions for limb salvage in patients older than 75 years with limb-threatening soft tissue sarcomas and other extremity tumors. Ann Surg Oncol. 2003;10:32-7.

14. Sonneveld EJ, Vrouenraets BC, van Geel BN, et al. Systemic toxicity after isolated limb perfusion with melphalan for melanoma. Eur J Surg Oncol. 1996;22:521-7.

15. Ariyan CE, Brady MS, Siegelbaum RH, et al. Robust antitumor responses result from local chemotherapy and CTLA-4 blockade. Cancer Immunol Res. 2018;6:189-200. 